\title{
Asymptotic giant branch evolution: Where do we stand?
}

\author{
Paola Marigo \\ Department of Physics and Astronomy Galileo Galilei, University of Padova, \\ Vicolo dell'Osservatorio 3, Padova, Italy \\ email: paola.marigo@unipd.it
}

\begin{abstract}
I provide a synthetic overview of the present status of stellar models for the asymptotic giant branch phase, one of the most complex and still uncertain stages of stellar evolution. In particular I will focus on two aspects that are most relevant in the context of the planetary nebulæ progeny, namely: the chemical composition of the AGB ejecta, and the mass of the bare $\mathrm{CO}$ core left after the ejection of the stellar mantle at the AGB tip. Recent progress, present uncertainties, and future perspectives to constrain AGB models are briefly discussed.
\end{abstract}

Keywords. Stars: AGB and post-AGB, stars: white dwarfs, stars: mass loss, stars: carbon

\section{Introduction}

In the framework of the stellar evolution theory, it is convenient to group stars in different ranges of initial masses depending on their final fate (see Herwig 2005). Stars with $M_{\mathrm{i}} \leqslant M_{\text {up }}$ develop an electron-degenerate CO core after central He-burning, then experience the canonical Asymptotic Giant Branch (AGB) phase and eventually leave $\mathrm{CO}$ white dwarfs as dark remnants. Stars with $M_{\mathrm{up}} \leqslant M_{\mathrm{i}} \leqslant M_{\text {mas }}$ are able to ignite carbon in mildly-degenerate conditions, leading to the formation of O-Ne-Mg cores. They will proceed through the so-called super-AGB phase (see the contribution by GarcíaBerro, this conference) ending as either ONe white dwarfs or electron-capture supernovæ, depending on the core mass. Both critical masses $M_{\text {up }}$ and $M_{\text {mas }}$ heavily depend on uncertain aspects of stellar models (e.g. treatment of convection and overshooting); in particular present predictions locate $M_{\text {up }}$ in the range $\sim 6-8 M_{\odot}$ (Siess 2007).

This contribution will mainly focus on the evolutionary features predicted for AGB stars evolved from progenitors with low and intermediate masses, $0.9 \lesssim M_{\mathrm{i}} / M_{\odot} \leqslant M_{\text {up }}$. The AGB evolution can be schematically divided into two phases, namely the early-AGB (E-AGB) and the Thermally-Pulsing AGB (TP-AGB). We refer to Herwig (2005) for a recent comprehensive review of this phase.

\subsection{Mixing episodes prior to the TP-AGB phase}

Important changes in the surface chemical composition may take place before the onset of the TP-AGB phase.

The first dredge-up. As the star ascends the red giant branch (RGB) it expands while the base of the convective envelope reaches into regions where partial H-burning has taken place at earlier stages. The first dredge-up determines the increase in the envelope abundances of ${ }^{13} \mathrm{C},{ }^{14} \mathrm{~N}$ and a decrease in the ${ }^{12} \mathrm{C}$ content (e.g. Forestini \& Charbonnel 1997; Marigo 2001).

Extra-mixing after the first dredge-up. Several spectroscopic observations ( $\mathrm{Li}$, $\left.{ }^{12} \mathrm{C} /{ }^{13} \mathrm{C}, \mathrm{N}, \mathrm{O}\right)$ in open and globular clusters of the Galaxy and the Magellanic Clouds provide compelling evidence that non canonical mixing starts as low-mass stars reach 


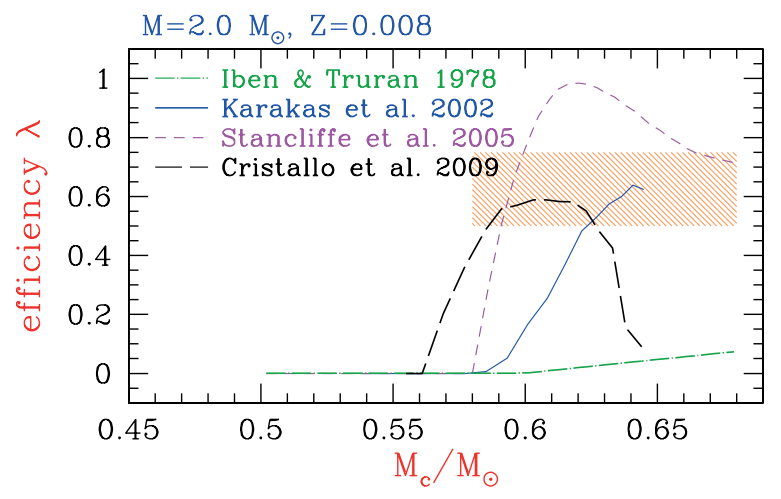

Figure 1. Efficiency of the third dredge-up, as a function of the core mass, predicted for a $2.0 M_{\odot}, Z=0.008$ model according to different authors. The dashed rectangular area brackets the typical values for $\lambda$ required to reproduce the observed carbon-star luminosity functions in the Magellanic Clouds, following the results of Groenewegen \& de Jong (1993); Stancliffe et al. (2005); Marigo \& Girardi (2007).

the bump in the luminosity function of the RGB. While various hypotheses were suggested in the past, thermohaline mixing has been recently identified as the actual mechanism able to explain the data (Charbonnel \& Lagarde (2010); Stancliffe et al. (2009), Denissenkov \& Pinsonneault (2008); Charbonnel \& Zahn (2007)).

The second dredge-up. Following central He-exhaustion the He-burning shell is established, and the star starts to evolve at increasing luminosity while approaching its Hayashi line for the second time during its life. During the initial stages, the so-called early-AGB (E-AGB), the H-shell is extinguished and the inner edge of the convective envelope moves inward. In stars more massive than $3.5-4.0 M_{\odot}$ the second dredge-up takes place, with notable effects both on the surface composition and internal structure of the star (e.g. Forestini \& Charbonnel 1997; Boothroyd \& Sackmann 1999). The products of complete H-burning are brought up to the surface, mainly ${ }^{4} \mathrm{He}$ and ${ }^{14} \mathrm{~N}$, while ${ }^{12} \mathrm{C}$ and ${ }^{16} \mathrm{O}$ are depleted at the surface. At the same time the mass coordinate of the $\mathrm{H}-$ exhausted core is significantly shifted inward, so that final core masses do not exceed $M_{\mathrm{c}} \simeq 1.0-1.1 M_{\odot}$, well below the Chandrasekhar limit of $1.4 M_{\odot}$.

\subsection{The TP-AGB phase}

This phase is characterized by the quasi-periodic occurrence of thermal pulses, corresponding to thermal instabilities of the He-burning shell. The surface chemical composition may be significantly altered by mixing events taking place at thermal pulses, the third dredge-up, and, in most massive AGB stars $\left(M>3.5-4.0 M_{\odot}\right)$ by hot-bottom burning, i.e. hydrogen-burning in the deepest and hottest layers of the convective envelope during quiescent stages.

The third dredge-up. During the third dredge-up the base of the convective envelope reaches across the extinct $\mathrm{H}$-shell and penetrates into the inter-shell region where the He-flash nucleosynthesis has taken place. The net consequence is the enrichment in the surface abundances of ${ }^{4} \mathrm{He}$, primary ${ }^{12} \mathrm{C}$ and ${ }^{16} \mathrm{O},{ }^{19} \mathrm{~F},{ }^{22} \mathrm{Ne},{ }^{25} \mathrm{Mg}$, and s-process elements (Iben \& Truran 1978; Cristallo et al. 2009). At the same time, the third dredge-up causes a reduction of the core mass, the entity of which is traditionally quantified via the efficiency parameter, $\lambda=\Delta M_{\mathrm{dred}} / \Delta M_{\mathrm{c}}$, given by the ratio between the dredged-up mass and the core mass increment over the previous quiescent inter-pulse period. Despite being a key quantity, the efficiency $\lambda$ and its dependence on stellar mass and metallicity are presently affected by large uncertainties, due to our still poor physical description of convection and mixing.

Figure 1 displays how predictions for $\lambda$ have changed over the years: while in the past very weak dredge-up characterizes low-mass AGB models, a fact designated by Iben (1981) as the "carbon star mystery" (see also Iben \& Truran 1978), present models 


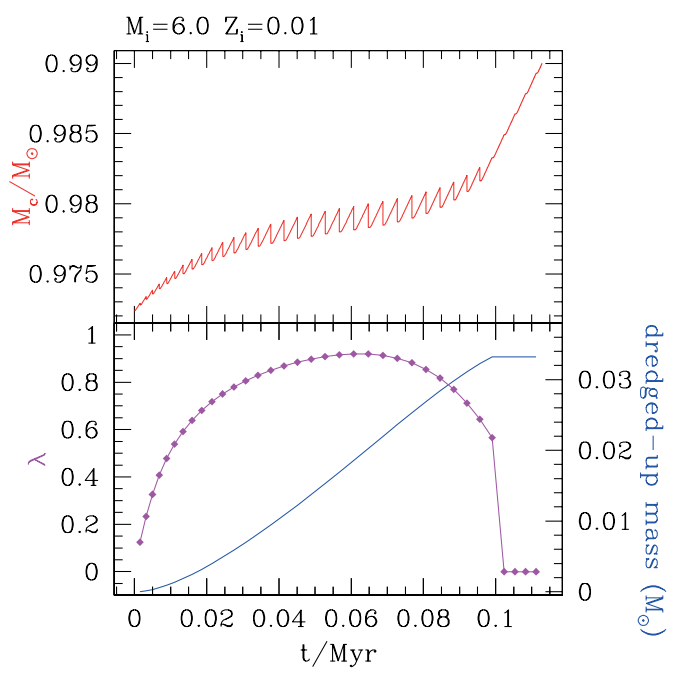

Figure 2. Top panel: Typical evolution of the core mass as a function of time during the whole TP-AGB evolution of an intermediate-mass experiencing very deep third dredge-up and HBB as well. Note the saw-tooth trend due to the periodic occurrence of the dredge-up events. Bottom panel: Evolution of the efficiency of the $3^{\text {rd }}$ dredge-up (from Karakas et al. 2002) and cumulative dredged-up mass.

predict larger $\lambda$, as required to reproduce observations of $\mathrm{C}$ stars in the Magellanic Clouds (Marigo et al. 1999; Marigo \& Girardi 2007). We also note that results may differ considerably from author to author, which reflects the critical dependence of the third dredge-up on technical and numerical details (see Frost \& Lattanzio (1996), Mowlavi (1999) for thorough analyses).

While $\lambda$ values for low-mass stars are still quite heterogeneous, there is a general agreement among authors in predicting $\lambda \approx 1$ for more massive AGB stars, with $M_{\mathrm{i}}>$ 3-4M $\odot$ (Karakas et al. 2002; Herwig 2004; Ventura \& D'Antona 2005; Weiss \& Ferguson 2009). A typical example is shown in Fig. 2. This implies a small increase, o even the nearly invariance of the core mass during the TP-AGB phase, so that the remnant mass would practically coincide with the core mass after the $2^{\text {nd }}$ dredge-up.

In any case the definitive assessment of the third dredge-up requires a more realistic description of convection; in this respect it is worth mentioning the valuable work by Herwig et al. (2006), who presented the first hydrodynamic multi-dimensional simulations of He-shell flash convection.

Hot-bottom burning. This process is expected to affect crucially the evolution of all AGB stars with large core masses, $M_{\mathrm{c}}>0.8 M_{\odot}$, and sufficiently massive envelopes, $M_{\mathrm{env}}=M-M_{\mathrm{c}}>2 M_{\odot}$. The minimum mass for the occurrence of $\mathrm{HBB}$ is a function of metallicity $Z$, so that lower $Z$ favors the onset of HBB at lower stellar masses (Ventura \& D'Antona 2005). HBB produces two main evolutionary effects, namely i) it makes the stars brighter than expected by the classical $M_{\mathrm{c}}-L$ relation (Blöcker \& Schönberner 1991), and ii) it enriches the surface chemical composition with nuclei synthesized by the $\mathrm{C}-\mathrm{N}-\mathrm{O}, \mathrm{Ne}-\mathrm{Na}$, and $\mathrm{Mg}-\mathrm{Al}$ cycles (Forestini \& Charbonnel 1997). More recently HBB nucleosynthesis has gained much interest since metal-poor massive (super-)AGB stars are considered among the most likely candidates to explain the chemical patterns of stars in globular clusters (e.g. the O-Na anti-correlation; Carretta et al. 2005).

Figure 3 shows the pre-flash quiescent luminosity as a function of the core mass for a few full stellar TP-AGB models of different mass and metallicity (Karakas et al. 2002). The most massive stars are characterized by the HBB over-luminosity, i.e. a steep luminosity increase above the $M_{\mathrm{c}}-L$ relation, which is eventually recovered as soon as the envelope mass is drastically reduced by stellar winds. Note that the $M_{\mathrm{i}}=6 Z=0.0001$ model is 


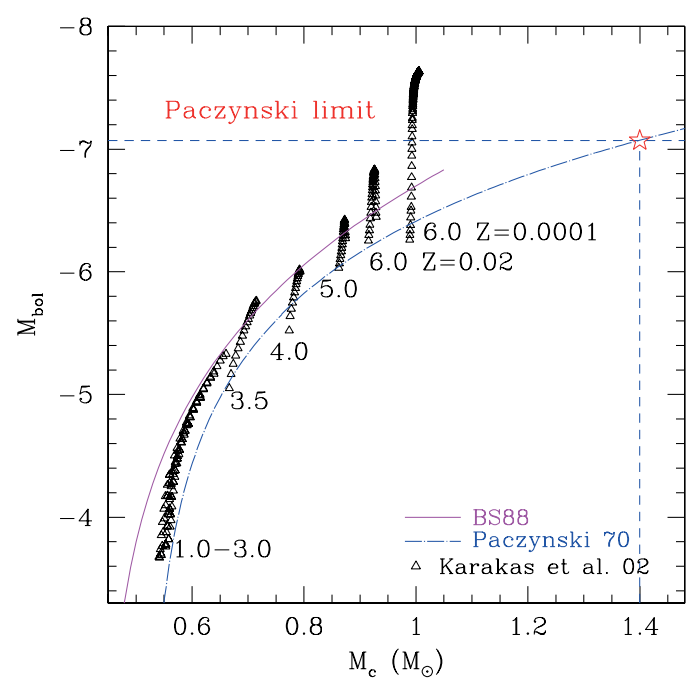

Figure 3. Luminosity as a function of the core mass along the TP-AGB. Sequences marked by triangles refer to the pre-flash luminosity maximum of TP-AGB stars with various initial masses, according to Karakas et al. (2002) full evolutionary calculations. Solid lines are fit $M_{\mathrm{c}}-L$ relations from Paczyński (1970) and Boothroyd \& Sackmann (1988).

expected to overcome the classical Paczynski limit $\dagger$, which in fact cannot be considered a physical limit anymore. HBB and third dredge-up interrelate with each other in a complex fashion: on one side $\mathrm{HBB}$ delays or even prevents the formation of massive $\mathrm{C}$ stars by converting the dredged-up carbon into nitrogen, on the other side the structural cooling caused by the enhanced C-rich opacity may weaken or even extinguish HBB (Marigo 2007; Ventura \& Marigo 2009, 2010).

\section{Molecular opacities}

Another fundamental issue for evolutionary AGB models is related to the adopted low-temperature opacities $(T<10000 \mathrm{~K})$. Until recently a common practice in most published TP-AGB evolutionary calculations is to use opacity tables that are strictly valid for scaled-solar abundances (e.g. Alexander \& Ferguson 1994), hence totally inadequate to produce realistic models for C stars. As already demonstrated by Marigo (2002); Marigo et al. (2003); Marigo \& Girardi (2007), the adoption of proper molecular opacities, consistently coupled with the surface composition, brings a radical improvement in the treatment of carbon-star evolutionary models, leading to solve several long-lasting discrepancies between theory and observations. For instance, the red tail drawn by field carbon stars in near-infrared colour-colour diagrams of the Magellanic Clouds (2MASS survey; see Marigo et al. (2003)) is reproduced, as well as the low $\mathrm{C} / \mathrm{O}$ and $T_{\text {eff }}$ values typically found in Galactic AGB C-type stars.

Figure 4 displays the sharp dichotomy in the molecular chemistry when the $\mathrm{C} / \mathrm{O}$ ratio passes from below to above unity (left panels), which has a dramatic effect on the evolution of the effective temperatures of AGB stars (right panel).

Molecular opacities for variable chemical mixtures have become available only recently (Lederer \& Aringer 2009; Helling \& Lucas 2009; Marigo \& Aringer 2009). The ÆSOPUS tool $\ddagger$ by Marigo \& Aringer (2009) allows the computation in real time of opacities for any set of elemental abundances. Recent full AGB calculations with C-rich opacities are

$\dagger$ Traditionally the Paczynski limit corresponds to the maximum luminosity that an AGB star complying with the $M_{\mathrm{c}}-L$ relation may reach when its core mass has grown up to the Chandrasekhar limit, $M_{\mathrm{c}} \simeq 1.4 M_{\odot}$

$\ddagger$ An interactive web-interface is available at http://stev.oapd.inaf.it/aesopus. 

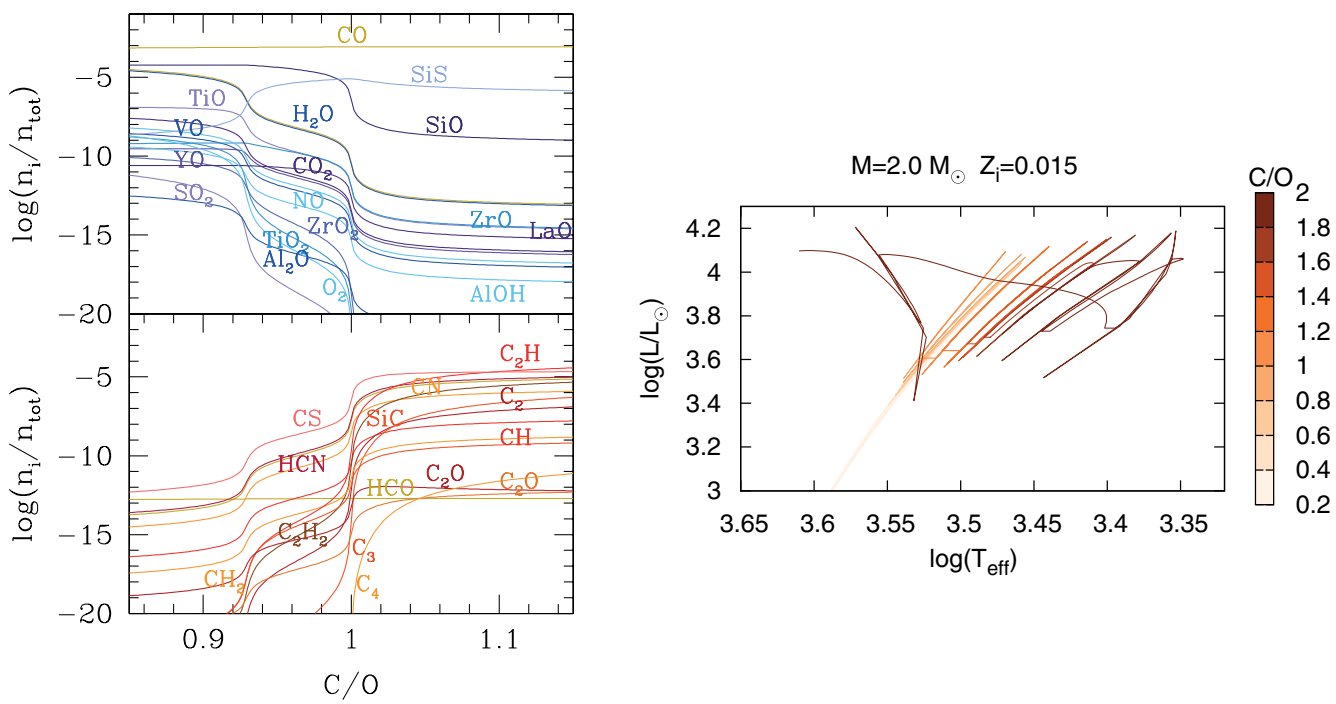

Figure 4. Left panel: molecular concentrations predicted with the ÆSOPUS tool (Marigo \& Aringer 2009) as a function of the $\mathrm{C} / \mathrm{O}$ ratio, assuming a gas mixture with $\log (T)=3.3, \log (\rho)=-11.1, Z_{\text {ref }}=0.02$. The increase of $\mathrm{C} / \mathrm{O}$ follows that of $\mathrm{C}$, while the $\mathrm{O}$ abundance is kept unchanged. The molecules are divided into two groups, namely: O-bearing molecules (top-left panel) and C-bearing molecules (bottom-left panel). Note the sharp change in molecular concentrations at $\mathrm{C} / \mathrm{O} \approx 1$. Right panel: Predicted TP-AGB evolutionary track for a star experiencing the third dredge-up. Molecular opacities are calculated on-the-fly with the ÆSOPUS (Marigo et al., in preparation).

presented by Cristallo et al. (2007); Weiss \& Ferguson (2009); Ventura \& Marigo (2009, 2010); Kamath et al. (2011).

\section{Chemical yields}

Several sets of chemical yields from low- and intermediate-mass stars, covering wide ranges of initial stellar masses and metallicities, are nowadays available (Karakas 2010; Cristallo et al. 2009; Ventura \& D'Antona 2009; Stancliffe \& Jeffery 2007; Izzard et al. 2004; Marigo 2001; Forestini \& Charbonnel 1997). While there is a general agreement on qualitative trends, significant quantitative differences do exist among the sets of chemical yields to different model prescriptions, in particular for mass loss, convection, and overshooting. In addition to the classical light elements ( $\mathrm{He}, \mathrm{C}, \mathrm{N}, \mathrm{O}, \mathrm{Ne}$ ) the enrichment in s-process elements, recently detected in some PNe, poses a critical challenge to models of AGB stars (see Karakas contribution, this conference).

\section{The initial - final mass relation}

The initial-final mass relation (IFMR) links the mass of a star on the main sequence, $M_{\mathrm{i}}$, with the remnant mass, $M_{\mathrm{f}}$, of the white dwarf (WD) left at the end of its evolution, It is the result of the complex interplay among different processes, such as dredge-up events and mass loss, and their dependence on stellar mass and metallicity. Even if it is impossible to disentangle the individual role of each factor involved, fig. 5 attempts to summarize a few general trends that can be extracted from AGB evolutionary models.

Dependence on the $3^{\text {rd }}$ dredge-up. The more efficient the $3^{\text {rd }}$ dredge-up, the lower is the effective increase of the core mass during the TP-AGB phase. In the extreme case $\lambda \simeq 1$, the final mass would correspond the core mass at the onset of the TP-AGB phase. The 

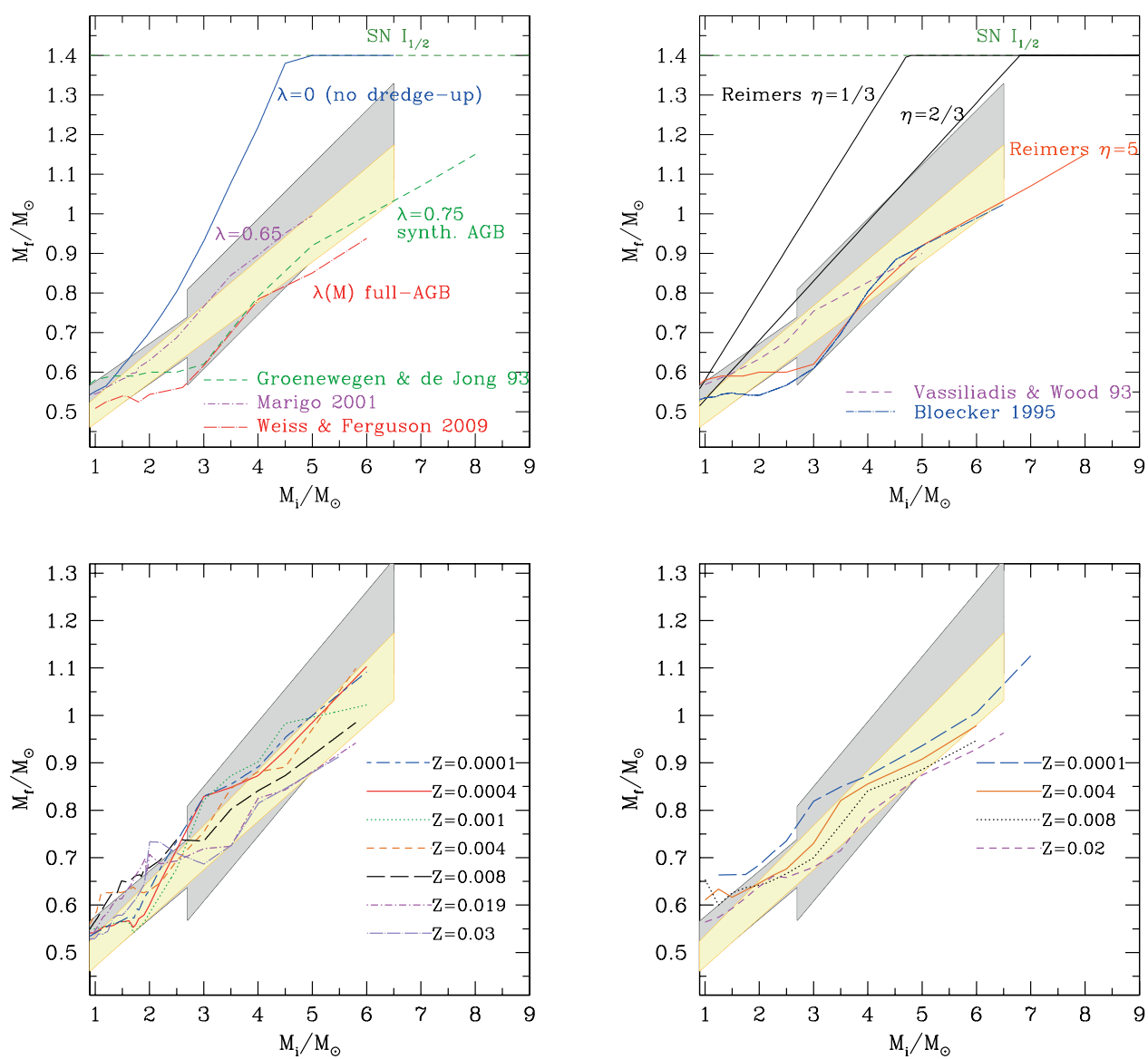

Figure 5. Dependence of the predicted IFMR on basic parameters, namely: efficiency of the third dredge-up (top left-hand side panel), the assumed mass-loss rates (top right-hand side panel), and metallicity (left bottom panel: Marigo \& Girardi (2007); right bottom panel: Karakas et al. (2002)). The shaded areas correspond to the semi-empirical IFMR from recent compilations (Ferrario et al. 2005; Catalán et al. 2008; Salaris et al. 2009).

semi-empirical IFMR seems to support a modest increase of the core mass during the TPAGB. In fact, current AGB models predict values of the core mass at the first thermal pulse which are already inside the observed width $\Delta M_{\mathrm{f}}$ of the semi-empirical IFMR (Karakas et al. 2002; Marigo \& Girardi 2007). Moreover, by assuming no dredge-up we expect that intermediate-mass stars would be able to reach the Chandrasekhar limit and thus explode as $\mathrm{SN} \mathrm{I}_{1 / 2}$, while the semi-empirical IFMR indicates that at $M_{i} \simeq 5-7 M_{\odot}$ the white dwarf masses are around $1 M_{\odot}$, in any case far from the limiting mass for $\mathrm{C}$ deflagration.

Dependence on mass loss. The larger the mass-loss rates, the shorter is the duration of the TP-AGB phase, hence the lower is the mass of the remnant. First of all, we note that simply extending to the AGB phase the classical Reimers law, commonly used (with a parameter $\eta \simeq 0.3-0.4$ ) for stars on the RGB, would again make intermediate-mass stars proceed through the $\mathrm{SN} \mathrm{I}_{1 / 2}$ channel (provided that the third dredge-up $\lambda<1$ ). Synthetic AGB models (Groenewegen \& de Jong 1993) have proved that $\eta \simeq 5$ would be required on the AGB in order to match both the observed IMFR and carbon star luminosity functions on the Magellanic Clouds. However, over the years, it has become 
clear that the Reimers law is not suitable to describe the efficiency of mass loss on the AGB, as this formula does not account for the observed steep increase of the massloss rate at increasing luminosity, as shown by studies of mass-losing pulsating AGB stars (Vassiliadis \& Wood 1993). Many other formalisms/recipes for AGB mass loss have been proposed, either semi-empirical (van Loon et al. 2005; Groenewegen et al. 1998; Vassiliadis \& Wood 1993), or derived from hydrodynamic dust-driven pulsation-assisted wind models (Mattsson et al. 2010; Wachter et al. 2008, 2002; Arndt et al. 1997; Bloecker 1995; Bowen \& Willson 1991; Bedijn 1988).

Dependence on metallicity. A quite robust result of stellar evolution is that stars enter the TP-AGB phase with core masses that are larger at decreasing metallicity $Z$, as a result of the previous central He-burning phase. How the subsequent TP-AGB evolution shapes the IFMR as a function of $Z$ is the result of the overall dependence on metallicity of all processes involved (e.g. mass loss efficiency, depth of $3^{\text {rd }}$ dredge-up, HBB overluminosity), which is still not robustly determined. As an example we show in Fig. 5 (bottom panles) two sets of predicted IFMRs for several metallicities, taken from the calculations by Marigo \& Girardi (2007) (left panel), and Karakas et al. (2002) (right panel). We see that while in the former set the dependence on $Z$ is not monotonic over the whole range of stellar masses, in the latter more massive white dwarfs are always expected at decreasing $Z$. This difference is likely due to differences in the description of mass loss in molecular opacities of $\mathrm{C}$ stars, coupled to the current surface $\mathrm{C} / \mathrm{O}$ in Marigo \& Girardi (2007), frozen to a scaled-solar mixture in Karakas et al. (2002).

\section{The need of population synthesis models to calibrate the AGB}

While significant improvements of AGB modelling have been achieved in the recent years (detailed calculations covering larger $Z$ and $M$ ranges; molecular chemistry and opacities, extended nuclear networks, extra-mixing, rotation, etc.), we are still facing large uncertainties mainly due to the currently deficient descriptions of convection and mass loss.

In this context a fundamental help can be provided by population synthesis models including AGB stars (Marigo et al. 2008). By a close comparison with many different observables (AGB star counts in clusters and galaxies, multi-band photometry and pulsation-period distributions of AGB variables, chemical abundances in PNe, dust mineralogy of enshrouded AGB stars, etc.) it would be possible to characterize the key processes of the AGB phase (third dredge-up, HBB, mass loss) by constraining the parameters that measure their efficiency as a function of stellar mass and metallicity. In this way AGB lifetimes and chemical yields, hence the actual role of AGB stars in galaxies could be eventually assessed.

\section{Acknowledgements}

P.M. acknowledges financial support from contract ASI-INAF n. I/009/10/0.

\section{References}

Arndt, T. U., Fleischer, A. J., \& Sedlmayr, E. 1997, A\&A, 327, 614

Bedijn, P. J. 1988, A\&A, 205, 105

Blöcker, T. \& Schönberner, D. 1991, A\& A, 244, L43

Bloecker, T. 1995, A\& A, 297, 727

Boothroyd, A. I. \& Sackmann, I.-J. 1988, ApJ, 328, 641

- 1999, ApJ, 510, 232 
Bowen, G. H. \& Willson, L. A. 1991, ApJL, 375, L53

Carretta, E., Gratton, R. G., Lucatello, S., Bragaglia, A., \& Bonifacio, P. 2005, A $E A$ A, 433, 597

Catalán, S., Isern, J., García-Berro, E., Ribas, I., Allende Prieto, C., \& Bonanos, A. Z. 2008, $A \mathscr{\ddots} A, 477,213$

Charbonnel, C. \& Lagarde, N. 2010, $A \& A$, 522, A10

Charbonnel, C. \& Zahn, J.-P. 2007, A\&A, 476, L29

Cristallo, S., Straniero, O., Gallino, R., Piersanti, L., Domínguez, I., \& Lederer, M. T. 2009, ApJ, 696, 797

Cristallo, S., Straniero, O., Lederer, M. T., \& Aringer, B. 2007, ApJ, 667, 489

Denissenkov, P. A. \& Pinsonneault, M. 2008, ApJ, 684, 626

Ferrario, L., Wickramasinghe, D., Liebert, J., \& Williams, K. A. 2005, MNRAS, 361, 1131

Forestini, M. \& Charbonnel, C. 1997, A\& $A S, 123,241$

Frost, C. A. \& Lattanzio, J. C. 1996, ApJ, 473, 383

Groenewegen, M. A. T. \& de Jong, T. 1993, A\&A, 267, 410

Groenewegen, M. A. T., Whitelock, P. A., Smith, C. H., \& Kerschbaum, F. 1998, MNRAS, 293, 18

Helling, C. \& Lucas, W. 2009, MNRAS, 398, 985

Herwig, F. 2004, ApJ, 605, 425

- 2005, ARAA, 43, 435

Herwig, F., Freytag, B., Hueckstaedt, R. M., \& Timmes, F. X. 2006, ApJ, 642, 1057

Iben, I., Jr. 1981, ApJ, 246, 278

Iben, I., Jr. \& Truran, J. W. 1978, ApJ, 220, 980

Izzard, R. G., Tout, C. A., Karakas, A. I., \& Pols, O. R. 2004, MNRAS, 350, 407

Kamath, D., Karakas, A. I., \& Wood, P. R. 2011, ArXiv e-prints. 1111.1722

Karakas, A. I. 2010, MNRAS, 403, 1413

Karakas, A. I., Lattanzio, J. C., \& Pols, O. R. 2002, PASA, 19, 515

Lederer, M. T. \& Aringer, B. 2009, A\& A, 494, 403

Marigo, P. 2001, $A \mathscr{E} A, 370,194$

$-2002, A \mathscr{E} A, 387,507$

- 2007, A\&A, 467, 1139

Marigo, P. \& Aringer, B. 2009, $A \mathscr{E} A, 508,1539$

Marigo, P. \& Girardi, L. 2007, $A \mathscr{E} A, 469,239$

Marigo, P., Girardi, L., \& Bressan, A. 1999, A\&A, 344, 123

Marigo, P., Girardi, L., Bressan, A., Groenewegen, M. A. T., Silva, L., \& Granato, G. L. 2008, $A \mathscr{E} A, 482,883$

Marigo, P., Girardi, L., \& Chiosi, C. 2003, A\& $A$, 403, 225

Mattsson, L., Wahlin, R., \& Höfner, S. 2010, A\& $A$, 509, A14+

Mowlavi, N. 1999, A\&SA, 344, 617

Paczyński, B. 1970, Acta Astronomica, 20, 287

Salaris, M., Serenelli, A., Weiss, A., \& Miller Bertolami, M. 2009, ApJ, 692, 1013

Siess, L. 2007, A\& A, 476, 893

Stancliffe, R. J., Church, R. P., Angelou, G. C., \& Lattanzio, J. C. 2009, MNRAS, 396, 2313

Stancliffe, R. J., Izzard, R. G., \& Tout, C. A. 2005, MNRAS, 356, L1

Stancliffe, R. J. \& Jeffery, C. S. 2007, MNRAS, 375, 1280

van Loon, J. T., Cioni, M.-R. L., Zijlstra, A. A., \& Loup, C. 2005, A\&A, 438, 273

Vassiliadis, E. \& Wood, P. R. 1993, ApJ, 413, 641

Ventura, P. \& D'Antona, F. 2005, A\&A A 431, 279

- 2009, A\& A, 499, 835

Ventura, P. \& Marigo, P. 2009, MNRAS, 399, L54

- 2010, MNRAS, 408, 2476

Wachter, A., Schröder, K.-P., Winters, J. M., Arndt, T. U., \& Sedlmayr, E. 2002, A\&্A, 384, 452

Wachter, A., Winters, J. M., Schröder, K.-P., \& Sedlmayr, E. 2008, A\&\&A, 486, 497

Weiss, A. \& Ferguson, J. W. 2009, A\& A, 508, 1343 